\title{
The Industrial Necessity of Leakage Current Verification Using Sm Doped Ceria Electrolytes in SOFCs and Future Applications
}

\author{
T. Miyashita*
}

1-6-3, Mitsuya-kita, Yodogawa-ku, Osaka, 532-0032, Japan

\begin{abstract}
The use of Sm-doped Ceria electrolytes (SDC) in SOFCs (solid oxide fuel cells) lowers the open circuit voltage $(\mathrm{OCV})$ below the Nernst voltage (Vth) obtained using Yttria-stabilized Zirconia (YSZ) electrolytes. The OCV is classically calculated with Wagner's equation. However, using the SDC electrolytes requires both qualitative and quantitative experimental verification of leakage currents. Furthermore, there are limitations in Wagner's equation, due to linear transport theory, when SDC electrolytes are used. The constant voltage loss without leakage current due to a mixed ionic and electronic conducting (MIEC) dense anode is proposed, and local equilibrium is considered to address the transition state during ion hopping. Only a carrier species having sufficient energy to overcome the activation energy can contribute to current conduction, which is described by a different constant in the definition of chemical potential and electrical potential. This difference explains results from the use of dense MIEC anodes. In this study, the industrial importance of this topic is discussed, and applications to future technologies are introduced.
\end{abstract}

Keywords: SOFC, water electrolysis, Ceria, MIEC, Wagner's equation.

\section{INTRODUCTION}

SOFCs directly convert the chemical energy of gases like hydrogen or methane into electrical energy. A solid oxide film, which is usually YSZ, is used as the electrolyte. In the electrolyte, oxygen ions serve as the main carriers. If the operating temperature (873-1273 K) could be lowered, the life span of the cells would be extended. To accomplish this, higher ion-conducting electrolyte materials such as SDC have been studied. However, the OCV of SDC cells is about $0.8 \mathrm{~V}$, which is lower than the Nernst voltage $(1.15 \mathrm{~V})$ for YSZ cells. The low OCV value is due to partial electronic conductivity under a hydrogen gas atmosphere at the anode. Wagner's equation $[1,2]$ also predicts this low value and is expressed as:

$O C V=\frac{R T}{4 F} \int_{p O_{2}^{\prime}}^{p O_{2}^{\prime \prime}} t_{\text {ion }} d \ln P O_{2}$

where F, R, T, tion, $p O_{2}^{\prime}$ and $p O_{2}^{\prime \prime}$ are Faraday's constant, the gas constant, the absolute temperature, the ionic transference number, and the oxygen partial pressures at the cathode and anode, respectively. Equation 1 is a classical equation that is still used for modern theoretical calculations [3] and is within the limits of linear transport theory. The current-voltage relation from Equation 1 was calculated using the constant field approximation [4].

However, qualitative [5] and quantitative [6] experimental verification of leakage currents using SDC electrolytes is necessary. Furthermore, there are limitations in Wagner's equation using SDC electrolytes, which come

*Address correspondence to this author at the 1-6-3, Mitsuya-kita, Yodogawa-ku, Osaka, 532-0032, Japan; Tel: 090-1204-1259;

Fax:+81-797-32-6171; E-mail: tom_miya@ballade.plala.or.jp from the limits of linear transport theory. The electric field near the cathode is large enough to lead to dielectric breakdown, which has never been reported, and the constant field approximation is not rational [7]. Consequently, a low OCV value should be possible without any leakage current.

Instead of Wagner's equation, local equilibrium can be used to describe the transition state during ion hopping, and constant voltage loss without leakage current due to the MIEC dense anode has been proposed [8]. From conventional theory [4], the current density I of the charge carrier " $\mathrm{k}$ " is:

$I_{k}=-\frac{\sigma_{k}}{Z_{k} F} \operatorname{grad} \eta_{k}$

where $\sigma_{k}, Z_{k}$, and $\eta_{k}$ are the conductivity, the charge number, and the electrochemical potential, respectively, of the charge carrier $\mathrm{k}$. Examples of $\mathrm{k}$ include electrons, holes, and ions. The electrochemical potential $\eta_{k}$ is expressed as:

$\eta_{\mathrm{k}}=\mu_{\mathrm{k}}+\mathrm{ZF} \phi$.

where $\mu \mathrm{k}$ and $\phi$ are the chemical potential and the electrical potential of the charge carrier k, respectively. Only a carrier species that has sufficient energy to overcome the activation energy can contribute to current conduction. Ions move from one vacancy to any other vacancy through the saddle point in the lattice structure. At the saddle point, the speed of hopping ions is zero and the electrical potential energy of hopping ions should be decreased by the electrical potential caused by the lattice atoms that are moved by hopping ions. Consequently, further transformations are necessary in Equation 3. Here, $\phi$ does not exist at the vacancies, but it exists at the saddle point:

$\mathrm{ZF} \phi=\mathrm{ZF} \phi-\mathrm{NEa}$ 
$\mu_{\mathrm{i}}=\mu_{\mathrm{i}}+\mathrm{NEa}$.

where N, Ea, and $\mu \mathrm{i}$ are Avogadro's number, the activation energy of oxygen ions, and the chemical potential of the ions, respectively. These transformations show that ZF $\phi$ is small compared to conventional values, the electrochemical potential does not change, and $\mu_{i}$ is increased in the electrolytes.

From Equation 4, including $(\mathrm{Z}=-2, \mathrm{~N} / \mathrm{F}=1 / \mathrm{e})$, we see:

$\phi=\phi-\mathrm{NEa} / \mathrm{ZF}=\phi+\mathrm{Ea} / 2 \mathrm{e}$.

Therefore, the value of $\phi$ is a constant, Ea/2e, even near the anode. When the ionic transference number is near unity at the cathodic side and sufficiently small at the anodic side, $\phi$ is neutralized by free electrons in the MIEC dense anode. This voltage loss becomes thermal energy and is recycled to activate the ions. Even if the ionic current (Ii) is very small, the $\mathrm{OCV}$ is written as:

$\mathrm{OCV}=\mathrm{V}$ th - RiIi - polarization voltage losses $-\mathrm{Ea} / 2 \mathrm{e}=\mathrm{V}$ th $\mathrm{Ea} / 2 \mathrm{e}$,

where $\mathrm{Ri}$ is ionic resistance. Equation 7 has previously been discovered empirically [9]. For example, the OCV using SDC electrolytes is $0.8 \mathrm{~V}$ at $1073 \mathrm{~K}(=1.15 \mathrm{~V}-0.7 \mathrm{eV} / 2 \mathrm{e})$. From Equation 7, the OCV is constant during electrode degradation, which was experimentally verified [5]. Consequently, the constant voltage loss without leakage current is supported by the empirical equation and experimental results.

Some problems have already been discovered in Equation 7. The OCV in Equation 7 becomes small using double or triple layered cells. For example, the measured $\mathrm{OCV}$ is $0.84 \mathrm{~V}$ for an electron blocking YSZ layer on the cathode side of an SDC electrolyte, which is larger than $0.8 \mathrm{~V}$ [10]. In order to solve this problem, an electric double layer formed between the contacts of two electrolytes is explained in the next section. Furthermore, Equation 7 can not be used when space charge effect can not be ignored.

The modification of the transport equations in solid state ionics is not an isolated topic that can be regarded as a minor research need. In the next section, the industrial necessity of leakage current verification using $\mathrm{Sm}$ doped Ceria electrolytes in SOFCs and future applications will be discussed.

\section{THE INDUSTRIAL NECESSITY OF LEAKAGE CURRENT VERIFICATION USING SM DOPED CERIA ELECTROLYTES IN SOFCS AND FUTURE APPLICATIONS}

\subsection{Basic Measurement Technology}

\subsubsection{Clarifying the Mechanism of Voltage Loss with MIEC Dense Anode Parts}

Voltage measurement is the most basic method in electrochemistry. Deviations of the experimental voltage from the theoretical voltage can only be attributed to the following explanations:

1. There are leakage currents.

2. The ion concentrations are different from expected values.
3. There is contamination from unknown ions.

Even if biochemical scientists discover every gene in DNA and every ion in the cell, they must give up clarifying the difference between the theoretical voltage and experimental voltage. In order to clarify the mechanism of voltage loss, the following statement must hold:

\section{"The voltage loss is caused by the MIEC dense anode layer without any leakage currents".}

Breakage of the ion channel by unknown reasons may be caused by electronic dielectric breakdown of the anode parts. For the biochemical researcher, it is impossible to note this fact without prior knowledge. The situation is shown in Fig. (1). Consequently, Equation 7 is important not only in the area of solid state ionics, but also in every area using MIEC materials.

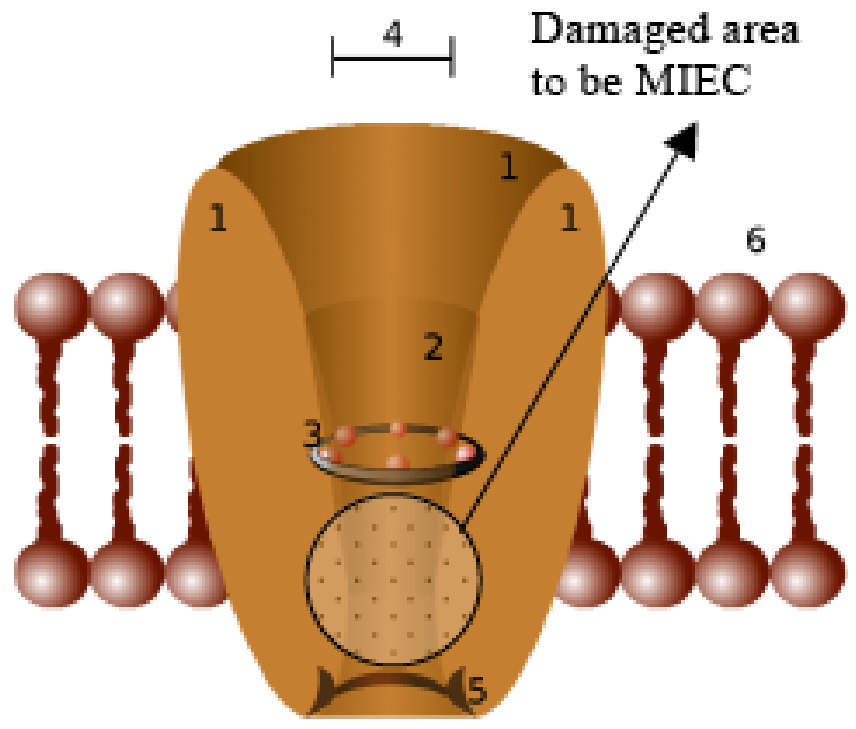

Fig. (1). Schematic diagram of an ion channel (from free Wikipedia, http://en.wikipedia.org/wiki/Ion_channel). 1 - channel domains (typically four per channel), 2 - outer vestibule, 3 selectivity filter, 4 - diameter of selectivity filter, 5 phosphorylation site, 6 - cell membrane.

The voltage between 1 and 5 becomes lower than the theoretical values when the anode parts become MIEC. Without MIEC knowledge, it is impossible to clarify the mechanism of the possible voltage loss.

\subsubsection{Measuring the Ionic Activation Energy of MIEC from the $\mathrm{OCV}$}

Using Equation 7, the ionic activation energy can be measured via the OCV. Some materials, like $\mathrm{YBa}_{2} \mathrm{Cu}_{3} \mathrm{O}_{7}-\delta$ (YBCO), have a very high electronic conductivity and anisotropy. When the Nernst voltage was $0.2 \mathrm{~V}$ with a YSZ layer on the cathode side (shown in Fig. 2), the OCV was only $0.05 \mathrm{~V}$ [11]. This gives a calculated ionic activation energy value of $0.3 \mathrm{eV}$. This is similar to the $0.38 \mathrm{eV}$ value given in [12].

It is very difficult to measure ionic activation energy of MIEC materials (like LSM or YBCO) that have electronic conductivity much higher than the ionic conductivity. Unfortunately, these MIEC materials are very weak in reducing environments, so it is difficult to observe clear 
results. But measuring the ionic activation energy of MIEC from the OCV is expected to be a very useful technology in the future.

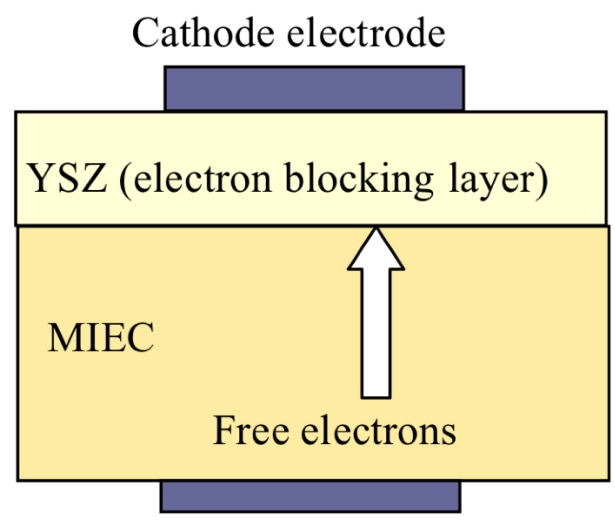

Anode electrode

Fig. (2). Measuring the ionic activation energy of MIEC from the OCV.

\subsection{SOFC Technology}

\subsubsection{Stack Failure Problem Using MIEC Electrolytes}

It is well known that cell imbalance can lead to battery failure [13]. This is more important in SDC cells than with YSZ electrolytes. Riess's model indicates that the OCV using SDC electrolytes will be changed after electrode degradation. According to Riess's model using SDC electrolytes, the stack failure cannot be avoided, since the OCV of each cell will change with time. Consequently, verification of leakage currents using $\mathrm{Sm}$ doped Ceria electrolytes in SOFCs is necessary before making the stacks. As the verification method, observing unchanged OCV during anode degradation becomes important [14].

\subsubsection{Cell Designs Using Dense MIEC Anode}

It is well accepted that the OCV can be increased by using a YSZ passivation film on the anode side [10]. The situation is shown in Fig. (3). However, this decreases the maximum power output by the large anode polarization voltage loss. So using a YSZ electron blocking layer on the cathode side has been tried (shown in Fig. 2), but the OCV remains at $0.84 \mathrm{~V}$. Since 1995, this problem has been explained as the limitation of the Hebb-Wagner polarization method [15]. But the OCV still cannot be raised in 2009 [10]. Consequently, the voltage loss in the OCV using a dense MIEC anode may not be caused by technological problems but for theoretical reasons, which can be seen from Equation 7. This is very important for cell designs. In the 1990s, some researchers wanted to develop a gastight dense MIEC anode layer and gave up due to the loss of time and money. Because current general knowledge is that anode porosity should be high, cell designs using dense MIEC anodes have been forgotten.

From Equation 7, using MIEC passivation materials that have small ionic activation energy can raise the OCV. For an $\mathrm{Ea}$ of $0.3 \mathrm{eV}$, the expected $\mathrm{OCV}$ is:

$\mathrm{OCV}=1.15 \mathrm{~V}-0.3 \mathrm{eV} / 2 \mathrm{e}=1.0 \mathrm{~V}$

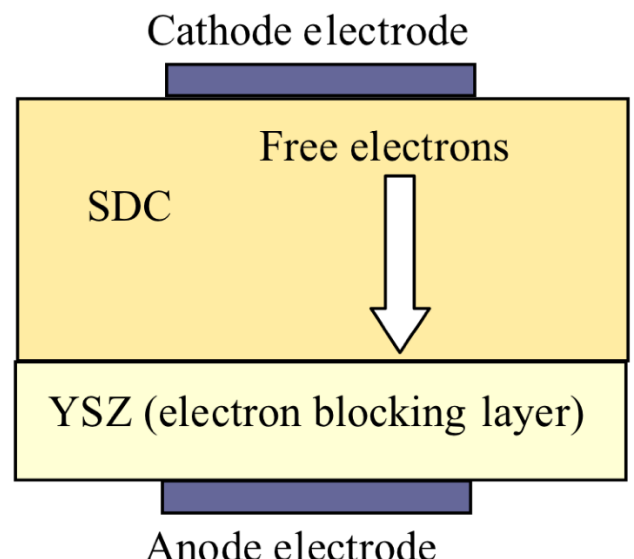

Fig. (3). SDC electrolyte with YSZ layer.

This situation is shown in Fig. (4), where the passivation film should not be an insulator for electrons. The anode performance is enhanced by the electron conductivity of the MIEC passivation layer.

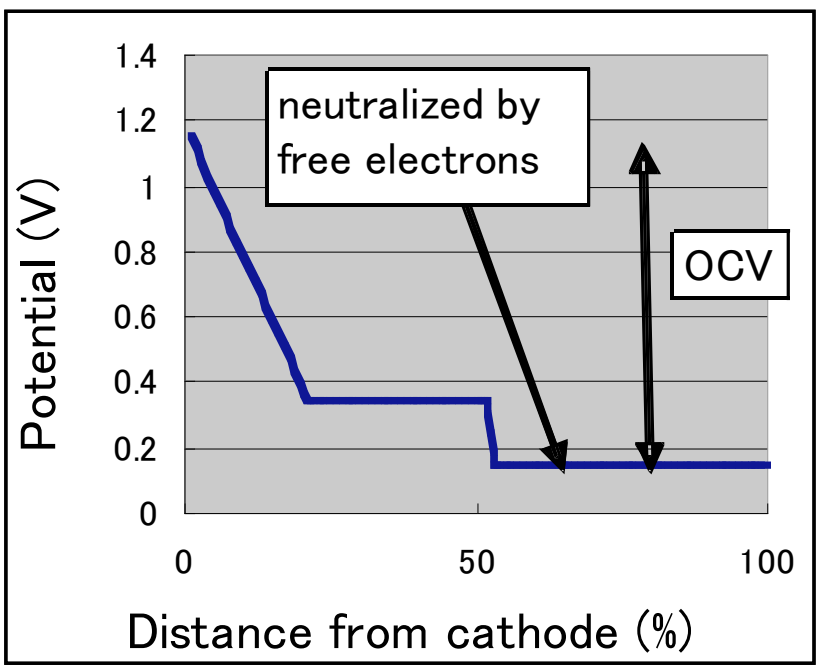

Fig. (4). Higher OCV values are obtained using small Ea, MIEC passivation films.

The OCV can be raised without an anode YSZ layer.

\subsubsection{Rectification of Oxygen Ions to Support Equation 7}

The OCV is about $0.8 \mathrm{~V}$ for a single SDC electrolyte. The OCV was $0.84 \mathrm{~V}$ for an electron blocking layer on the cathode side of an SDC electrolyte [10]. If there are no leakage currents in the doped Ceria electrolytes, the OCV cannot increase. This seems to be contrary to Equation 7. But this problem can be solved by considering the rectification of oxygen ions.

The $0.04 \mathrm{~V}(=0.84-0.8)$ should be from the rectification of oxygen ions. At the point of contact of an YSZ and SDC electrolyte, there should be an electric double layer. This means that oxygen ions can easily move from YSZ to SDC. The following situations consider two different ionic conductors. Ions with large chemical potentials move more easily than ions with small chemical potentials. This is because entropy decreases with increased chemical potential when the enthalpy is the same. In order to increase the total 
entropy, ions must move from electrolyte that has larger ionic activation energy to smaller ionic activation energy. The existence of double electric layer can be made sure measuring the OCV of an electron blocking YSZ layer on the anode side of an SDC electrolyte $(1.11 \mathrm{~V}=1.15 \mathrm{~V}$ 0.04V). This is shown in Fig. (5).

Thus, rectification can be controlled by the following equation:

Ea1 $($ cathode $)>\mathrm{Ea} 2$ (anode)

Here, Ea1 and Ea2 are the activation energies of the ionic conductors. For a SOFC, the cathode material usually has an ionic activation energy of $1.6 \mathrm{eV}$, which is larger than that of electrolytes. So there are not any problems, but Equation 9 is not satisfied for SDC electrolytes with an anode YSZ layer. Serious degradation problems have been reported for the contact of two electrolytes [16]. This type of cell is developed by many researchers, so it is important to ensure the rectification of oxygen ions.

\subsection{Water Electrolysis Technology}

Water electrolysis may be more important than SOFCs. Producing cheap hydrogen gas is an important future goal; however, these technologies are years from practical application. Equation 9 is one of necessity. The cathode materials (for example, LSM) of SOFCs usually have ionic activation energy of $1.6 \mathrm{eV}$, which is larger than that of electrolytes. However, for water electrolysis, Ea2 should be less than that of the electrolytes. Consequently, the cathode materials in an SOFC cannot be used as anode materials for water electrolysis. From this aspect, electrolytes that have small ionic activation energy have greater potential for real applications [17].

\subsection{Super Conducting Oxides}

The mechanism for super conducting oxides is still unknown. If the mechanism of super conducting oxides is elucidated, research in this area will develop very quickly.
In MIEC materials, ions lose energy during hopping after traveling the saddle point, since free electrons gain energy. The value of energy loss during one hop is equal to the ionic activation energy. So the energy of hopping ions becomes very small near the end of the hop. It means that ions can be colder than electrolytes while hopping after traveling the saddle point until the end of the hop. The situation is shown in Fig. (6). If hopping ions are cold enough, according to BCS theory, they can be centers of superconductivity.

The rate for supplying energy to ions is proportional to the electronic resistance of the electrolytes, which is related to the thermal conductivity in the electrolytes and difficult to calculate. But under a superconducting situation, hopping ions are thermally isolated, since there should be no resistance for ions. So there is no balance between the gain and loss of hopping ion energy under a superconducting situation. The rate of the total energy loss of the ions can be calculated from the ionic activation energy, the number of ions hopping, and the amount of hops per second, which are related to the lattice frequency. The number of cold oxygen ions cannot be ignored. The calculated result is shown in Appendix.

The importance and relevance of data was supported by the weak oxygen isotope effect and electron-phonon interactions [18]. Consequently, the concept of cold hopping ions that can be the center of superconductivity is worth detailed research.

\section{DISCUSSIONS}

In this study, the industrial necessity of leakage current verification using Sm doped Ceria electrolytes in SOFCs is explained. This topic is not an isolated and minor topic, but of vital importance to the scientific community. Several future technological applications are also introduced. A further detailed and quantitative explanation may be needed; however, the industrial implications of these technologies are too great to dismiss based on their incomplete understanding.
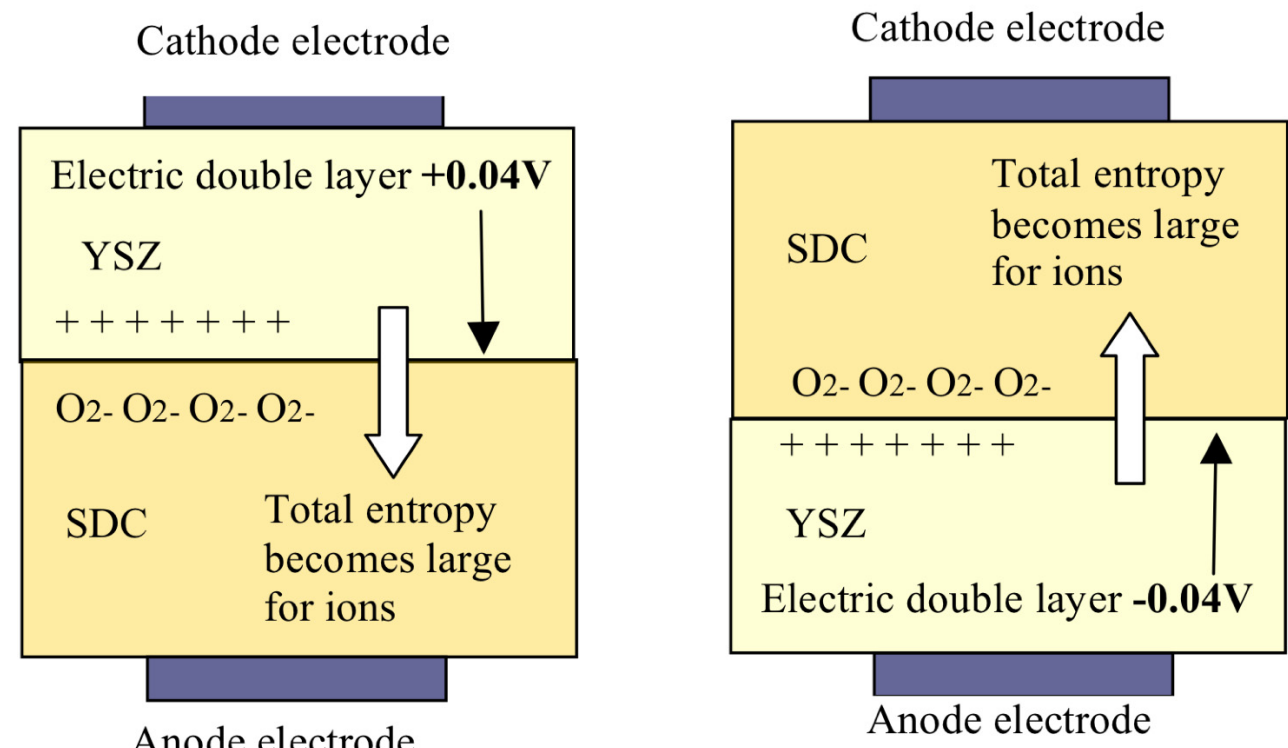

Fig. (5). Explanation of rectification between the contacts of two electrolytes. 

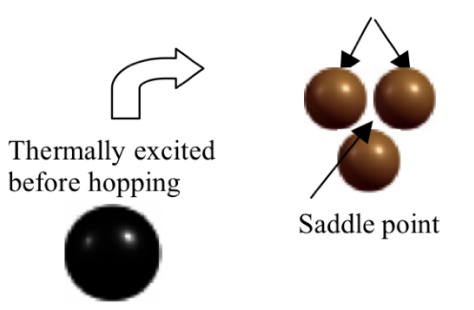

Fig. (6). Ions can be colder than MIEC electrolytes after hopping.

There remain strong suspicions in Equation 7. It is not the main purpose of this report, but making more detailed scientific descriptions is absolutely needed.

The classical thermodynamics have been strongly believed to be completely finished. This belief is shown in the words of Richard Feynman who is the one of the most famous scientist in the 20th century. "There are not any fundamental discoveries in the classical thermodynamics".

It is not true. Ions having sufficient energy to overcome the activation energy become carriers which can escape from electrolyte. But we cannot separate the Boltzmann distribution using passive filters which is well known as "Maxell's demon". According to the second law of thermodynamics, Maxwell's demon should be denied. Using the information theory, Maxwell's demon was denied by Landauer's Principle. But in the detail mechanism of denying Maxwell's demon is not clarified thermodynamically still yet. It means that there remains the fundamental discovery in the classical thermodynamics still yet.

Only a carrier species having sufficient energy to overcome the activation energy can contribute to current conduction, which is described by a different constant in the definition of chemical potential and electrical potential. This difference explains results from the use of dense MIEC anodes. Thus, the effective Gibbs energy is calculated from Equation 7. But in the explanation of the effective Gibbs energy from the fundamental level, there should be the definition of the forth law of thermodynamics which should be described not only in the area of solid state ionics.

\section{APPENDIX}

The symbol list is shown in Table 1. The rate of total energy loss of the ions can be calculated as follows. The purpose of this calculation is to determine whether the rate can be ignored or not.

Table1. Symbol List

\begin{tabular}{|c|c|c|}
\hline Symbol & Meaning & Value \\
\hline \hline $\mathrm{T}$ & Absolute temperature & $93 \mathrm{~K}$ (for example) \\
\hline $\mathrm{Ea}$ & Ionic activation energy & $0.3 \mathrm{eV}$ (for example) \\
\hline $\mathrm{eV}$ & Energy unit & $1.6 \mathrm{E}-19 \mathrm{~J}$ \\
\hline $\mathrm{k}$ & Boltzmann constant & $1.38 \mathrm{E}-23$ \\
\hline $\mathrm{f}$ & Lattice vibration & $1 \mathrm{E}+13 \mathrm{~Hz}$ \\
\hline $\mathrm{n}$ & number of ions & unknown \\
\hline
\end{tabular}

Potential Energy transfer

to free electrons in MIEC
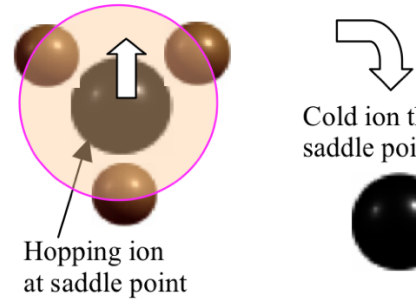

Cold ion though out saddle point

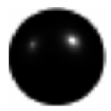

At first,

$$
k T=1.38 \times 10^{-23} \times 93 /\left(1.6 \times 10^{-19}\right)=0.008 \mathrm{eV}
$$

The number of hopping ions per second $(\mathrm{N})$ is;

$$
\begin{aligned}
N= & \exp (-E a / k T) \times f \times n=\exp (-0.3 / 0.008) \\
& \times 10^{13} \times n=0.00052 \times n
\end{aligned}
$$

Energy loss by hopping ions per second (Eloss) is;

$$
E_{\text {loss }}=N \times E a=0.00052 \times n \times 0.3 \mathrm{eV}=0.00016 \mathrm{eV} \times n
$$

Thermal energy of ions (Ethermal)

$$
E_{\text {thermal }}=3 \mathrm{kT} \times n=0.008 \times n=0.024 \mathrm{eV} \times n
$$

So,

$$
\frac{E_{\text {loss }}}{E_{\text {thermal }}}=\frac{0.00016 \mathrm{eV}}{0.024 \mathrm{eV}}=0.007
$$

From Equation 14, ions lose $0.7 \%$ of their thermal energy per second. It means that $0.7 \%$ of ions become very cold in one second. This is not negligible.

\section{REFERENCES}

[1] Wagner C. Beitrag zur Theorie des Anlaufvorgangs. Z Phys Chem 1933; B41: 42.

[2] Rickert H. Electrochemistry of Solids - An Introduction. Springer, Berlin, Heidelberg 1982; p. 99.

[3] Zha SW, Xia CR, Meng GY. Calculation of the e.m.f. of solid oxide fuel cells. J Appl Electrochem 2001; 31: 93-8.

[4] Riess I. Current-voltage relation and charge distribution in mixed ionic electronic solid conductors. J Phys Chem Solids 1986; 47: 129-38.

[5] Miyashita T. Necessity of verification of leakage currents using Sm doped Ceria electrolytes in SOFCs. J Mater Sci 2006; 41:3183-4.

[6] Lai W, Haile SM. Electrochemical impedance spectroscopy of mixed conductors under a chemical potential gradient: A case study of Pt|SDC|BSCF. Phys Chem Chem Phys 2008; 10: 865-83.

[7] Miyashita T. Theoretical verification necessity of leakage currents using sm doped ceria electrolytes in SOFCs. Open Mater Sci J 2009; 3: 33-9.

[8] Miyashita T. Loss of Gibbs energy using sm-doped ceria electrolytes in sofcs considering local equilibrium while ion hopping. Open Mater Sci J 2009; 3: 47-9.

[9] Miyashita T. Empirical equation about open circuit voltage in SOFC. J Mater Sci 2005; 40: 6027-7

[10] Lima Hyung-Tae, Virkar Anil V. Measurement of oxygen chemical potential in Gd2O3-doped ceria-Y2O3-stabilized zirconia bi-layer electrolyte, anode-supported solid oxide fuel cells. J Power Sources 2009; 192: 267-78.

[11] Jak MJG, Riess I, Schoonman J. Combination of ac-impedance spectroscopy and short circuiting measurements applied on YBa2Cu3O7 - $\delta$. Solid State Ionics 1996; 86-88: 1409-13. 
[12] Zhang H, Du K, Ye H. Electric resistivity and its relaxation due to the variation of the oxygen content in a ceramic $\mathrm{Y} 2 \mathrm{BaCuOx}$. Supercond Sci Technol 2003; 16: 479-83.

[13] Hyung-Tae L, Virkar Anil V. A study of solid oxide fuel cell stack failure by inducing abnormal behavior in a single cell test. J Power Sources 2008; 185: 790-800.

[14] Guo W, Liu J, Zhang Y. Electrical and stability performance of anode-supported solid oxide fuel cell with strontium- and magnesium-doped lanthanum gallate thin electrolyte. Electrochim Acta 2008; 53: 4420-7.

[15] Riess I. Review of the limitation of the Hebb-Wagner polarization method for measuring partial conductivities in mixed ionic electronic conductors. Solid State Ionics 1996; 91; 221-32.
Zhang X, Gazzarri J, Robertsona M, Decès-Petit C, Kesler O. Stability study of cermet-supported solid oxide fuel cells with bi-layered electrolyte. J Power Sources 2008; 185: 1049-55.

[17] Zhu B, Albinsson I, Andersson C, Borsand K, Nilsson M, Mellander B. Electrolysis studies based on ceria-based composites. Electrochem Commun 2006; 8: 495-8.

[18] Sasagawa T, Lanzara A, Gweon G-H, Zhou S, Graf J, Suryadijaya, Takagi H. Oxygen isotope effect on electron dynamics in Bi2Sr2CaCu2Oy: angle-resolved photoemission spectroscopy. Phys C: Superconduct 2005; 426-431: 436-40.

(C) T. Miyashita; Licensee Bentham Open.

This is an open access article licensed under the terms of the Creative Commons Attribution Non-Commercial License (http://creativecommons.org/licenses/by-nc/ 3.0/) which permits unrestricted, non-commercial use, distribution and reproduction in any medium, provided the work is properly cited 